\title{
MULTI-OBJECTIVE OPTIMIZATION LINE-UP ALGORITHM APPLIED TO THE DESIGN OF A NONLINEAR VIBRATION ABSORBER
}

\author{
R. A. Borges ${ }^{1}$, F. S. Lobato ${ }^{2}$, V. Steffen $\mathrm{Jr}^{3}$
}

${ }^{1}$ Departament of Mathematics, Federal University of Goiás, Av. Lamartine P. Avelar, 1120, Catalão, GO, zip code 75704-020, Brazil, romes.borges@ catalao.ufg.br

${ }^{2}$ Laboratory of Modeling, Simulation, Control and Optimization of Process, School of Chemical Engineering, fslobato@feq.ufu.br

${ }^{3}$ Laboratory of Mechanics and Structures (LMEst), School of Mechanical Engineering, vsteffen@mecanica.ufu.br, Federal University of Uberlândia, Av. João Naves de Ávila, 2121, Uberlândia, MG, ZIP CODE 38400-902, Brazil

\begin{abstract}
Discrete dynamic vibration absorbers (DVAs) are mechanical devices designed to attenuate the vibration level of different structures and machines. They have been used in several engineering applications, such as ships, power lines, aeronautic structures, civil engineering constructions subjected to seismic induced excitations, among other applications. Traditionally, different approaches based on optimization methods have been proposed to design dynamic vibration absorbers in the mono-objective context. In the present contribution a multi-objective optimization strategy based on the Line-up algorithm is proposed, associated with the Pareto dominance criterion and the crowding distance operator. The test-case analyzed in this work focuses on the theoretical study and numerical simulations of a two degree-of-freedom nonlinear damped system, constituted of a primary mass attached to the ground by a linear spring and the secondary mass attached to the primary system by a nonlinear spring (nDVA). The objectives are both to maximize the attenuation bandwidth and to minimize the amplitude of the system. The results indicate that the proposed approach characterizes an interesting alternative for multi-objective optimization problems as compared with other evolutionary strategies.
\end{abstract}

Keywords: Discrete dynamic vibration absorbers, multi-objective optimization, Line-up algorithm.

\section{INTRODUCTION}

Naturally, real-world problems involve the simultaneous optimization of two or more (often conflicting) objectives. The solution of the multi-objective optimization problem (MOOP) is different from that of a single-objective optimization problem. The main differ- 
ence is that multi-objective optimization problems normally have not one but a set of solutions that are all equally good [1].

Traditionally, the treatment of such problems is done by transforming the original MOOP into a mono-objective problem. However, the development of specific methodologies allows the formulation of the optimization problem in a way that various objectives can be taken into account simultaneously. In addition, as a number of points that constitutes the optimal solution are found, it is possible to explore these solutions according to the practical application studied [1]. In the literature, several methods for solving MOOP can be found [1]. These methods follow a preference-based approach, in which a relative preference vector is used to scalarize multiple objectives. Since classical searching and optimization methods use a point-by-point approach so that the solution is successively modified, the outcome of this classical optimization method is a single optimized solution. However, Evolutionary Algorithms (EA) can find multiple optimal solutions in one single simulation run due to their population-based search approach. Thus, EA are ideally suited for multi-objective optimization problems. A detailed account of multi-objective optimization using EA and some of the applications using genetic algorithms can be widely found in the literature [1].

In the modern engineering context, the use of discrete dynamic vibration absorbers (DVAs) have an important application to reduce noise and vibration levels in various types of systems such as compressors, robots, ships, power lines, airplanes, helicopters, among others [2]. In the last two decades, a great deal of effort has been devoted to the development of mathematical models for characterizing the mechanical behavior of nonlinear dynamic vibration absorbers (nDVAs) accounting for their typical dependence on parameters that control the nonlinearities. A particular type of nDVA is the so called viscoelastic neutralizer as studied in [3]. Different techniques have been proposed to design viscoelastic vibration absorbers, as shown in [4] and [5]. Despite the known complexity in modeling the nonlinear dynamics involved in this type of absorber, some general methodologies have proved to be appropriate to treat this type of problem. This aspect makes them very attractive for the modeling of nonlinear dynamic vibration absorbers. Among these strategies, it should be mentioned the theoretical study proposed by Pai [6] and Schulz [7], in which techniques to improve the stability and efficiency of nDVAs into a frequency band of interest have been proposed, leading to refined nDVAs. Also, Rice [8] and Shaw [9] suggested optimization strategies to be applied to the design of nDVAs by applying an asymmetric nonlinear Duffing-type element incorporated in the suspension for narrow-band absorption applications.

The present work focuses the numerical optimization of a two degree-of-freedom nonlinear damped system, constituted of a primary mass attached to the ground by a linear spring and the secondary mass attached to the primary system by a nonlinear spring (nDVA) using the Line-up algorithm [10], associated with the following operators: non-dominated sorting and crowding distance. The objectives are both to maximize the bandwidth and to minimize the amplitude of the system. This work is organized as follows. The mathematical formulation of the non-linear dynamic system is presented in Section 2. A review of multi-objective optimization and the Line-up algorithm are presented in Section 3. The results and discussion are described in Section 4. Finally, the conclusions and suggestions for future work conclude the paper. 


\section{MATHEMATICAL MODELING OF THE NON-LINEAR DYNAMIC SYSTEM}

Consider the vibratory system represented by the two degree-of-freedom model [11] shown in Fig. 1.

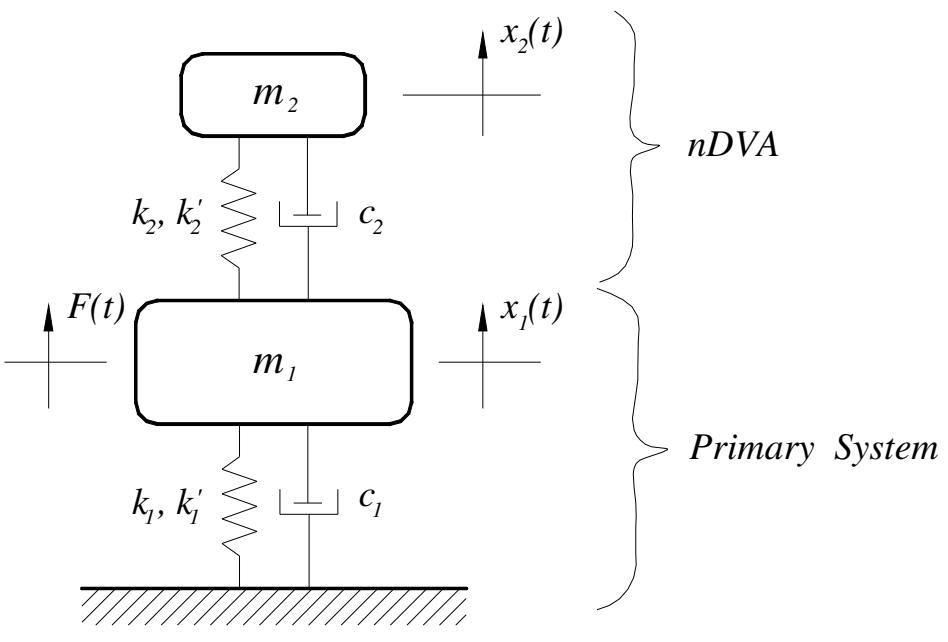

Figure 1 - Two degree-of-freedom non-linear mechanical system.

The interest is focused on frequency domain responses. In this case, to calculate the steady-state harmonic responses in the frequency domain the following relation for the external force $f(t)=f_{0} \cos (\omega t)$ is assumed (where $\omega$ is the excitation frequency). The constitutive forces of the springs are given as follows:

$$
s_{i}\left(x_{i}\right)=k_{i} x_{i}+k_{i}^{\prime} x_{i}^{3}, \quad i=1,2
$$

where $x_{1}$ and $x_{2}$ represent, respectively, the displacement of the primary system with respect to the ground and the displacement of the nDVA with respect to the primary mass. In the present case, the dampers are linear and the springs have nonlinear characteristics, where $k_{i}$ and $k_{i}^{\prime}$ indicate, respectively, their linear and nonlinear coefficients. By applying the Newton's second law, the following equations of motion of the nonlinear system are obtained:

$$
\left\{\begin{array}{l}
\left(m_{1}+m_{2}\right) \ddot{x}_{1}+m_{2} \ddot{x}_{2}+c_{1} \dot{x}_{1}+k_{1} x_{1}=f_{0} \cos (\omega t)-k_{1}^{\prime} x_{1}^{3} \\
m_{2} \ddot{x}_{1}+m_{2} \ddot{x}_{2}+c_{2} \dot{x}_{2}+k_{2} x_{2}=-k_{2}^{\prime} x_{2}^{3}
\end{array}\right.
$$

Aiming at obtaining the dimensionless normalized equations of motion for the nonlinear dynamic system, the displacements are normalized according to the following relation $y_{i}=x_{i} / x_{c}$, where $x_{c}=f_{0} / k_{1}$ and $i=1,2$. In addition, the following expressons are introduced: $\zeta_{1}=c_{1} /\left(2 \sqrt{k_{1} m_{1}}\right), \zeta_{2}=c_{2} /\left(2 \sqrt{k_{2} m_{2}}\right), \varepsilon_{1}=k_{1}^{n l} x_{c}^{2} /\left(m_{1} \omega^{2}\right), \varepsilon_{2}=k_{2}^{n l} x_{c}^{2} /\left(m_{2} \omega^{2}\right)$, $\mu=m_{2} / m_{1}, \quad F=f_{0} /\left(m_{1} x_{c} \omega^{2}\right), \quad \bar{\omega}_{1}^{2}=k_{1} / m_{1}, \bar{\omega}_{2}^{2}=k_{2} / m_{2}, \quad \omega_{1}=\bar{\omega}_{1} / \omega, \quad$ and $\omega_{2}=\bar{\omega}_{2} / \omega$. After manipulations, the following matrix form of the normalized equations is obtained:

$$
\mathbf{M} \ddot{\mathbf{y}}(t)+\mathbf{C} \dot{\mathbf{y}}(t)+\mathbf{K y}(t)=\boldsymbol{F}(t)
$$


where the normalized mass, damping and stiffness matrices are expressed, respectively, as:

$$
\mathbf{M}=\left[\begin{array}{cc}
1+\mu & \mu \\
\mu & \mu
\end{array}\right], \quad \mathbf{C}=\left[\begin{array}{cc}
2 \zeta_{1} \omega_{1} & 0 \\
0 & \mu 2 \zeta_{2} \omega_{2}
\end{array}\right], \quad \mathbf{K}=\left[\begin{array}{cc}
\omega_{1}^{2} & 0 \\
0 & \mu \omega_{2}^{2}
\end{array}\right]
$$

and the normalized displacement and force vectors are given, respectively, as follows:

$$
\boldsymbol{y}(t)=\left\{\begin{array}{l}
y_{1}(t) \\
y_{2}(t)
\end{array}\right\}, \boldsymbol{F}(t)=\left\{\begin{array}{c}
F \cos (\omega t)-\varepsilon_{1} y_{1}^{3} \\
-\mu \varepsilon_{2} y_{2}^{3}
\end{array}\right\}
$$

\subsection{Steady-state harmonic responses of the nonlinear dynamic system.}

Various perturbation methods are based on averaging. This means that the unknown functions of the problem are considered as dependent variables by using a shift of variables from the original dependent variable [12], [13]. These methods encompass techniques such as the following [14]: Krylov-Bogoliubov method, Krylov-Bogoliubov-Mitropolsky method, and the method of the generalized average. In the present case, the Krylov-Bogoliubov method will be used to integrate Eq. (3), leading to an approximate solution of the nonlinear differential equations of motion. Within this context, the Van der Pol Transformation [15], represented by expressions (6) and (7), are used to guarantee that the transformation is unique.

$$
\begin{gathered}
\mathbf{y}(\tau)=\mathbf{u}(\tau) \cos \tau+\mathbf{v}(\tau) \sin \tau \\
\dot{\mathbf{y}}(\tau)=-\boldsymbol{u}(\tau) \sin \tau+\boldsymbol{v}(\tau) \cos \tau
\end{gathered}
$$

where $\boldsymbol{u}=\left(u_{1}, u_{2}\right)^{T}$ and $\boldsymbol{v}=\left(v_{1}, v_{2}\right)^{T}$ are assumed to be slow functions of the normalized time $\tau=\omega t$.

After mathematical manipulation, we obtain a nonlinear algebraic system composed by four equations and four variables $\left(u_{1}, u_{2}, v_{1}, v_{2}\right)$ :

$$
\left\{\begin{array}{l}
\left(1+\mu-\omega_{1}^{2}\right) u_{1}+\mu u_{2}-2 \zeta_{1} \omega_{1} v_{1}-\frac{3}{4} \varepsilon_{1} u_{1}\left(u_{1}^{2}+v_{1}^{2}\right)+F=0 \\
\mu u_{1}+\left(\mu-\mu \omega_{2}^{2}\right) u_{2}-\mu 2 \zeta_{2} \omega_{2} v_{2}-\frac{3}{4} \mu \varepsilon_{2} u_{2}\left(u_{2}^{2}+v_{2}^{2}\right)=0 \\
\left(\omega_{1}^{2}-1-\mu\right) v_{1}-\mu v_{2}-2 \zeta_{1} \omega_{1} u_{1}+\frac{3}{4} \varepsilon_{1} v_{1}\left(u_{1}^{2}+v_{1}^{2}\right)=0 \\
\mu v_{1}+\left(\mu-\mu \omega_{2}^{2}\right) v_{2}+\mu 2 \zeta_{2} \omega_{2} u_{2}-\frac{3}{4} \mu \varepsilon_{2} v_{2}\left(u_{2}^{2}+v_{2}^{2}\right)=0
\end{array}\right.
$$

Then, the obtained values of the parameters $\left(u_{1}, u_{2}, v_{1}, v_{2}\right)$ are used to calculate the amplitudes of vibrations of the nonlinear system shown in Fig. 1, by performing the following relations $r_{1}=\sqrt{u_{1}^{2}+v_{1}^{2}}$ (for the primary mass) and $r_{2}=\sqrt{u_{2}^{2}+v_{2}^{2}}$ (for the nDVA). Moreover, in the numerical computations, the following parameters of force and frequency are also considered: $\beta=F / \omega_{1}^{2}$ (force parameter) and $\Omega=\omega / \bar{\omega}_{1}$ (frequency parameter), so that in equations (8) one has $\omega_{1}=1 / \Omega$ and $\omega_{2}=\rho / \Omega$, where $\rho=\omega_{2} / \omega_{1}$ (frequency ratio). 


\section{SENSITIVITY ANALYSIS OF STRUCTURAL RESPONSES}

In a mechanical system the parameters of mass, stiffness and damping establish the dependence with respect to a set of design parameters, which include physical and geometrical characteristics and the parameters that control the nonlinearities [16]. Such functional dependence can be expressed in a general form as follows:

$$
r=r(M(p), C(p), K(p))
$$

where $\boldsymbol{r}$ and $\boldsymbol{p}$ designate vectors of structural responses and design parameters, respectively.

The sensitivity of the structural responses with respect to a given parameter $\boldsymbol{p}_{i}$, evaluated for a given set of values of the design parameter $\boldsymbol{p}^{\mathbf{0}}$ is defined as the following partial derivative:

$$
\begin{aligned}
& \left.\frac{\partial \boldsymbol{r}}{\partial \mathrm{p}_{\mathrm{i}}}\right|_{\mathrm{p}^{0}}= \\
& \lim _{\Delta \mathrm{p}_{\mathrm{i}} \rightarrow 0}\left[\frac{\boldsymbol{r}\left(\boldsymbol{M}\left(\mathrm{p}_{\mathrm{i}}^{0}+\Delta \mathrm{p}_{\mathrm{i}}\right), \boldsymbol{C}\left(\mathrm{p}_{\mathrm{i}}^{0}+\Delta \mathrm{p}_{\mathrm{i}}\right), \boldsymbol{K}\left(\mathrm{p}_{\mathrm{i}}^{0}+\Delta \mathrm{p}_{\mathrm{i}}\right)\right)}{\Delta \mathrm{p}_{\mathrm{i}}}-\frac{\boldsymbol{r}\left(\boldsymbol{M}\left(\mathrm{p}_{\mathrm{i}}^{0}\right), \boldsymbol{C}\left(\mathrm{p}_{\mathrm{i}}^{0}\right), \boldsymbol{K}\left(\mathrm{p}_{\mathrm{i}}^{0}\right)\right)}{\Delta \mathrm{p}_{\mathrm{i}}}\right]
\end{aligned}
$$

where $\Delta p_{i}$ is an arbitrary variation applied to the current value of parameter $\boldsymbol{p}_{\boldsymbol{i}}{ }^{\mathbf{0}}$, while all other parameters remain unchanged. The sensitivity with respect to $\boldsymbol{p}_{i}$ can be estimated by finite differences by computing successively the responses corresponding to $\boldsymbol{p}_{i}=\boldsymbol{p}_{\boldsymbol{i}}{ }^{\mathbf{0}}$ and $\boldsymbol{p}_{\boldsymbol{i}}=\boldsymbol{p}_{\boldsymbol{i}}{ }^{\mathbf{}}+\Delta$ $p_{i}$.

Such procedure is an estimated approach enabling to calculate the sensitivity of the dynamic system responses with respect to small modifications introduced in the design parameters. Moreover, the results depend upon the choice of the value of the parameter increment $\Delta \boldsymbol{p}_{i}$. Another strategy consists in computing the analytical derivatives, as possible, of the structural responses with respect to the parameters of interest. This approach is not considered herein because of the numerical procedures used to solve the nonlinear equations.

\section{MULTI-OBJECTIVE OPTIMIZATION LINE-UP ALGORITHM}

\subsection{Multi-objective Optimization}

When dealing with MOOP, the notion of optimality needs to be extended. The most common one in the current literature is the one that was originally proposed in [17] and was later generalized by Pareto [18]. This notion is called Edgeworth-Pareto optimality, or simply Pareto optimality, and refers to finding good tradeoffs among all the objectives. This definition leads us to find a set of solutions that is called the Pareto optimal set, whose corresponding elements are called non-dominated or non-inferior solutions. The concept of optimality in a single objective optimization is not directly applicable in MOOPs. For this reason a classification of the solutions is introduced in terms of Pareto optimality, according to the following definitions [1]:

- Definition 1: The Multi-objective Optimization Problem (MOOP) can be defined as:

$$
f(x)=\left(f_{1}(x), f_{2}(x), \ldots, f_{m}(x)\right), m=1, \ldots, M
$$


subject to

$$
\begin{aligned}
h(x) & =\left(h_{1}(x), h_{2}(x), \ldots, h_{i}(x)\right), i=1, \ldots, H \\
g(x) & =\left(g_{1}(x), g_{2}(x), \ldots, g_{j}(x)\right), j=1, \ldots, J \\
x & =\left(x_{1}, x_{2}, \ldots, x_{n}\right), n=1, \ldots, N, x \in X
\end{aligned}
$$

where $x$ is the vector of design (or decision) variables, $f$ is the vector of objective functions and $X$ is denoted as the design (or decision) space. The constraints $h$ and $g(\geq 0)$ determine the feasible region.

- Definition 2: Pareto Dominance: For any two decision vectors $u$ and $v, u$ is said to dominate $v$, if $u$ is not worse than $v$ in all objectives and $u$ is strictly better than $v$ in at least one objective.

- Definition 3: Pareto Optimality: When the set $P$ is the entire search space, or $P=S$, the resulting non-dominated set $P^{\prime}$ is called the Pareto-optimal set. Like global and local optimal solutions in the case of single-objective optimization, there could be global and local Pareto-optimal sets in multi-objective optimization.

- Definition 4: Non-dominated Set: Among a set of solutions $P$, the non-dominated set of solutions $P$ ' are those that are not dominated by any member of the set $P$.

\subsection{Line-up Algorithm}

The Line-up algorithm aims at approximating the global optimum of a nonlinear objective function consisting of $N$ continuous decision variables where the only constraints that are taken into account are the restrictions of the decision variables regarding upper and lower limits. This algorithm starts with a population of $L$ possible solutions, which are selected by assuming a uniform probability distribution for each variable. In some cases we can use an initial guess that is believed to be located close to the optimum. Basically, the idea is to line up the solutions in each generation in a descending order, according to the corresponding values of the objective function. The crossover and mutation operations are then applied to the solutions as follows: during the crossover operation, for each adjacent pair the difference vector is computed, weighted by a random number between 0 and 1 and added to the first vector of the pair. The produced solution replaces the first vector if it produces an objective function value that is lower than the fitness value of the second vector. At the end of the crossover operation, the solutions are lined up again. Then the mutation operation is applied by taking into account that the worst members of the population should be altered substantially, while only small changes should be made in the best solutions. In order to achieve this, a different probability of mutation is calculated for each solution in the list, which is reduced from the top to the bottom. This probability defines the number of variables in each solution that will undergo the mutation operation. The reason for using non-uniform mutation is because it adds to the algorithm more local search capabilities. Using this approach, in the first iterations the variables that are mutated can be located anywhere in the input space. As the algorithm 
proceeds, more conservative moves are preferred and thus, search is concentrated on a more local level.

A pseudo-code description of the Line-up algorithm has the following steps (where itermax is the maximum number of iterations and iter is the current iteration):

(1) Generate a population of $L$ solutions $x_{i}, i=1, \ldots, L$, where the values for each decision variable are chosen randomly between the respective lower and upper bounds, assuming a uniform distribution.

(2) Increase the number of iterations by 1 , iter $=$ iter +1 (iteration current).

$L$.

(3) Compute the objective function value corresponding to each solution $f\left(x_{i}\right), i=1, \ldots$,

(4) Arrange the solutions so that they formulate a line in a descending order: $x_{1}, x_{2}, \ldots$, $x_{L}$ where $x_{i}$ precedes $x_{j}$ if $f\left(x_{i}\right)>f\left(x_{j}\right), i, j=1, \ldots, L$.

(5) Apply the crossover operator:

FOR $i=1, \ldots, L-1$

$x_{i, \text { new }}=x_{i}+r\left(x_{i+1}-x_{i}\right)$, where $r$ is a random number between 0 and 1

if $f\left(x_{i, \text { new }}\right)<f\left(x_{i+1}\right)$ then $x_{i}=x_{i, \text { new }}$

END

(6) Arrange the solutions so that they formulate a line in a descending order: $x_{1}, x_{2}, \ldots$, $x_{L}$ where $x_{i}$ precedes $x_{j}$ if $f\left(x_{i}\right)>f\left(x_{j}\right), i, j=1, \ldots, L$.

(7) Apply the non-uniform mutation operation:

$$
\begin{aligned}
& \text { FOR } i=1, \ldots, L \\
& p_{m, i}=(L-i+1) / L \\
& \text { FOR } j=1, \ldots, N \\
& \text { generate a random number between } 0 \text { and } 1 . \\
& \quad \text { IF } r<p_{m, i} \\
& \quad \text { generate a binary number and a random number } r \text { between } 0 \text { and } 1 . \\
& \quad \text { IF } b=0 \text { THEN } \mathrm{x}_{i, \text { new }}(j)=\mathrm{x}_{\mathrm{i}}(j)+\left(\mathrm{x}^{\mathrm{U}}(j)-\mathrm{x}_{i}(j)\right) r \exp (-2 \text { iter/itermax }) \text { END } \\
& \quad \text { IF } b=1 \text { THEN } \mathrm{x}_{i, \text { new }}(j)=\mathrm{x}_{\mathrm{i}}(j)-\left(\mathrm{x}_{i}(j)-\mathrm{x}^{\mathrm{L}}(j)\right) r \exp (-2 \text { iter/itermax }) \text { END } \\
& \text { END } \\
& \text { END } \\
& \text { IF } f\left(\mathrm{x}_{i, \text { new }}\right)<f\left(\mathrm{x}_{i+1}\right) \text { THEN } \mathrm{x}_{i}=\mathrm{x}_{i, \text { new }} \text { END }
\end{aligned}
$$

(8) Replace the solution corresponding to the maximum objective function with the best solution found so far and increase the number of iterations by 1 .

(9) If the number of iterations is equal to the maximum number of iterations max iter STOP. Otherwise go to step 2.

The Line-up algorithm has been successfully applied to various fields such as mathematical function optimization [19], [20]; separation process optimization [21]; optimal control problems [22], [23], among other applications.

\subsection{Multi-objective Optimization Line-up Algorithm}

Due to the success obtained by the Line-up algorithm in different applications in science and engineering, some attempts to extend this approach to solve multi-objective prob- 
lems can be found in the literature. In this work, the MOLup (Multi-objective Optimization Line-up) is presented. This approach is based on the canonical Line-Up algorithm associated with the non-dominated sorting and crowding distance operators. Basically, the proposed strategy has the following structure: an initial population (P1) of size NP is randomly generated. All dominated solutions are removed from the population through the non-dominated sorting operator. In this way, the population is sorted into non-dominated fronts $F_{j}$ (sets of vectors that are non-dominated with respect to each other). This procedure is repeated until each vector is a member of a front. A new population (P2) with $N P$ candidate is generated from the basic operations in the canonical Line-Up. Starting from population P3 ( $\{\mathrm{P} 1 ; \mathrm{P} 2\})$ of size $2 \times N P$, the classification is performed according to the dominance criterion and only the first $N P$ candidates are considered to form the non-dominated population (P4). If the number of individuals of the population $\mathrm{P} 4$ is larger than a number defined by the user it is truncated according to the criterion named the crowding distance operator [1]. This operator describes the density of solutions surrounding a vector. To compute the crowding distance for a set of population members the vectors are sorted according to their objective function value for each objective function. To the vectors with the smallest or largest values an infinite crowding distance (or an arbitrarily large number for practical purposes) is assigned. For all other vectors the crowding distance is calculated according to:

$$
\operatorname{dist}_{x_{i}}=\sum_{j=0}^{m-1} \frac{f_{j, i+1}-f_{j, i-1}}{\left|f_{j, \max }-f_{j, \min }\right|}
$$

where $f_{j}$ corresponds to the $j$-th objective function and $m$ equals the number of objective functions.

\section{RESULTS AND DISCUSSION}

In this work both the maximization of the attenuation bandwidth and the minimization of the amplitude of the two degree-of-freedom nonlinear damped system are considered. As previously mentioned, the system is constituted of a primary mass attached to the ground by a linear spring and a secondary mass attached to the primary system by a nonlinear spring (nDVA). For design purposes the following steps are established:

- Objective functions: maximization of the bandwidth and minimization of the amplitude.

- Design variables (normalized structural parameters): $0.9 \leq \rho \leq 1.1,0.045 \leq \mu \leq 0.055$, $0.09 \leq \beta \leq 0.11$ and $0.009 \leq \varepsilon_{2} \leq 0.011$.

- To solve the optimization problem the following heuristics are used for comparison purposes: Genetic Algorithm (NSGA) parameters [1]: population size (50), type of selection (normal geometric in the range [0 0.08$]$ ), type of crossover Arithmetic, 2), type of mutation (non-uniform [2 100 3]); Differential Evolution (MODE) parameters [24]: population size (50), perturbation rate and crossover probability both equal to 0.8 and DE/rand/1/bin strategy; and Line-up Algorithm parameters: population size (50).

- Stopping criterion: maximum number of generations (150). 
- Each algorithm was run 10 times by using 10 different seeds for the random generation of the initial population.

- To solve the non-linear equation system, the Newton Method is used.

Figure 1 present the Pareto's Curve considering all evolutionary strategies.

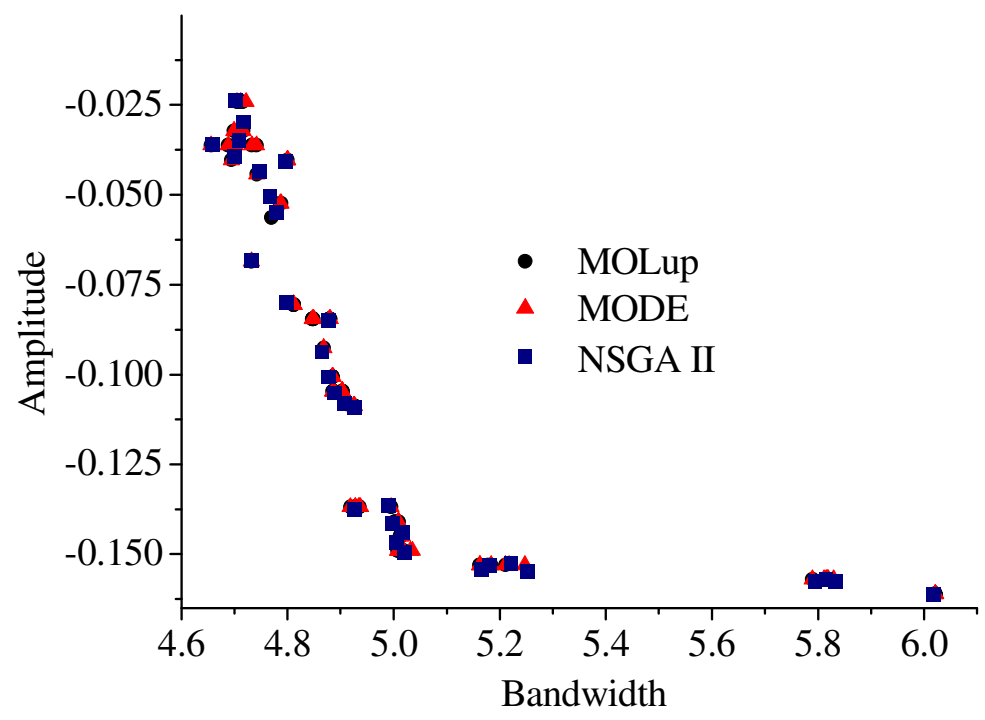

Figure 1. Pareto's Curve.

In this figure it is possible to observe that all evolutionary strategies considered in the present analysis are able to estimate satisfactory the Pareto's Curve, resulting a similar number of objective function evaluations for all the techniques conveyed.

Table 1 present some points ("best" value in terms of the maximization of the bandwidth and "best" value in terms of the minimization of the amplitude) of the Pareto's Curve obtained by the Line-up algorithm.

Table1. Some points of the Pareto's Curve.

\begin{tabular}{cccccc}
\hline$\rho$ & $\mu$ & $\beta$ & $\varepsilon_{2}$ & Bandwidth & -Amplitude \\
\hline 0.900 & 0.053 & 0.090 & 0.009 & 4.655 & -0.0362 \\
1.098 & 0.055 & 0.110 & 0.011 & 6.020 & -0.1611 \\
\hline
\end{tabular}

\section{CONCLUSIONS}

In this work the numerical optimization of a two degree-of-freedom nonlinear damped system composed of a primary mass attached to the ground by a linear spring and a secondary mass attached to the primary system by a nonlinear spring was studied. The system design was oriented so that both the maximization of the attenuation frequency bandwidth and the minimization of the amplitude response were taken into account. For this aim, a strategy based on the Line-up algorithm associated with the non-dominated sorting and crowding distance operators was proposed to obtain the optimal design of the system.

The numerical applications showed that the sensitivities of dynamic responses convey valuable information about the influence of the design parameters on the dynamic behavior of 
the nonlinear structure, being also a useful tool for design and analysis of modified systems and structural optimization. The choice of the design variables was based on previous knowledge regarding their sensitivities with respect to the amplitude peak and suppression bandwidth. It is worth mentioning that these parameters are directly associated with the effectiveness of the nDVA.

As demonstrated by the results, the nonlinearity factor is an important parameter to be investigated during the design procedure of nonlinear dynamic vibration absorbers, due to its contribution to the reduction of the vibration level. This point motivates an important procedure regarding the presented methodology, namely to obtain the optimal spring nonlinear coefficient that guarantees the best stable solution for a given system.

Finally, the results obtained show that the methodology used represents an interesting approach to the treatment of the formulated optimization problem as compared with other evolutionary strategies.

\section{Acknowledgements}

The authors acknowledge the financial support provided by FAPEMIG and CNPq (INCTEIE).

\section{REFERENCES}

[1] Deb K., "Multi-Objective Optimization using Evolutionary Algorithms", John Wiley \& Sons, Chichester, UK, ISBN 0-471-87339-X, 2001.

[2] Steffen Jr V., Rade D. A., "Dynamic Vibration Absorber", Encyclopedia of Vibration, Academic Press, ISBN 0-12-227085-1, 9-26, 2001.

[3] Espíndola J. J., Bavastri C. A., "Viscoelastic Neutralisers in Vibration Abatement: a Nonlinear Optimization Approach", Revista Brasileira de Ciências Mecânicas, XIX, 2, 154163, 1997.

[4] Espíndola J. J., Pereira P., Bavastri C. A., "Design of Optimum Systems of Viscoelastic Vibration Absorbers for a Given Material based on the Fractional Calculus Model", Journal of Vibration and Control, 14, 1607-1630, 2008.

[5] Espíndola J. J., Pereira P., Bavastri C.A., "Design of Optimum System of Viscoelastic Vibration Absorbers with a Frobenius Norm Objective Function", Journal of the Brazilian Society of Mechanical Sciences and Engineering, 31, 210-219, 2009.

[6] Nissen J. C., Popp K., Schmalhorst B., "Optimization of a Nonlinear Dynamic Vibration Absorber", Journal of Sound and Vibration, 99, 1, 149-154, 1985.

[7] Pai P. F., Schulz M. J., “A Refined Nonlinear Vibration Absorber”, International Journal of Mechanical Sciences, 42, 537-560, 1998.

[8] Rice H. J., McCraith J. R., "Practical Nonlinear Vibration Absorber Design”, Journal of 
Sound and Vibration, 116, 545-559, 1987.

[9] Shaw J., Shaw S. W., Haddow A. G., "On the Response of the Nonlinear Vibration Absorber", International Journal of Non-linear Mechanics, 24, 281-293, 1989.

[10] Yan L. X., Ph.D. dissertation, University of Chemical Technology, Beijing, 1998.

[11] Borges R. A., Lima A. M. G., Steffen Jr V.; "Robust Optimal Design of a Nonlinear Dynamic Vibration Absorber Combining Sensitivity Analysis", Shock and Vibration, 17 2010, 507-520, DOI 10.3233/sav-2010-0544.

[12] Nayfeh A. H., "Perturbation Methods", John Wiley \& Sons, Inc., 2000.

[13] Nissen J. C., Popp K., Schmalhorst B., "Optimization of a Nonlinear Dynamic Vibration Absorber", Journal of Sound and Vibration, 99, 1, 149-154. 1985.

[14] Thomsen J. J., "Vibrations and Stability", Springer-Verlag, $2^{\text {nd }}$ Edition, 2003.

[15] Zhu S. J., Zheng Y. F., Fu Y. M., “Analysis of Non-linear Dynamics of a Two Degreeof-Freedom Vibration System with Non-linear Damping and Non-linear Spring”, Journal of Sound and Vibration, 271, 2, 15-24, 1992.

[16] Haug E. J., Choi K. K., Komkov V., "Design Sensitivity Analysis of Structural Systems", Academic Press, 1986.

[17] Edgeworth F. Y., "Mathematical Psychics”, P. Keagan, London, England, 1881.

[18] Pareto V., "Manuale di Economia Politica", Societa Editrice Libraria, Milano, Italy. Translated into English by A.S. Schwier as Manual of Political Economy, Macmillan, New York, 1971.

[19] Yan L., Ma D., "Global Optimization of Non-convex Nonlinear Programs using Lineup Competition Algorithm", Computers and Chemical Engineering, 25, 1601-1610, 2001.

[20] Sarimveis H., Nikolakopoulos A., "A Line Up Evolutionary Algorithm for Solving Nonlinear Constrained Optimization Problems", Computers \& Operations Research, 32, 1499-1514, 2005.

[21] Yan L., Wei D., Ma D., "Line-up Competition Algorithm for Separation Sequence Synthesis", Process Systems Engineering, B. Chen and A.W. Westerberg (editors), Published by Elsevier Science B.V, 2003.

[22] Sun D., "The Solution of Singular Optimal Control Problems using the Modified Lineup Competition Algorithm with Region-Relaxing Strategy", ISA Transactions, 49, 106$113,2010$.

[23] Sun D., Huang T., "The Solutions of Time-Delayed Optimal Control Problems by the 
use of Modified Line-up Competition Algorithm", Journal of the Taiwan Institute of Chemical Engineers, 41, 54-66, 2010.

[24] Lobato F. S., "Multi-objective Optimization to Engineering System Design", PhD Thesis, School of Mechanical Engineering, Universidade Federal de Uberlândia, Brazil (in Portuguese), 2008. 